\title{
Modeling for Distribution of Compressive Stress from Loading Platens in Diametrical Compression Test
}

\author{
Yukari Higashi ${ }^{1}$, Shinnosuke Yoshinaga ${ }^{1}$, Rini Asnida Abdullah ${ }^{2}$ \& Takashi Tsutsumi $^{1 *}$ \\ ${ }^{1}$ Advanced Course of Civil Engineering, National Institute of Technology, Kagoshima College, \\ Kirishima, Kagoshima, 899-5193, Japan \\ ${ }^{2}$ Department of Civil Engineering, Universiti Teknologi Malaysia, 81310 Johor Bahru, Malaysia
}

\begin{abstract}
Diametrical Compression test is one of indirect tests to investigate the tensile strength of brittle materials. This test is spread because of easiness to perform. However, results from this test are not stable. Traditionally, calculations for this test are performed under a pair of opposite concentrated loading on the diameter of specimen. However, contact areas appear just before fracture of specimen in the fact and the stress distribution from loading platens is not obvious. It seems be one of reasons why the result from this test is not stable. In this study, the attempt to establish the modeling for distribution on loading platens in the diametrical compression test is shown. The aim of this modeling is to calculate stresses and displacement of specimen using complex stress function by Lekhnitskii. Therefore, this model is expanded in Fourier expansion. Accuracy of calculation using Fourier expansion depends on the number of terms. Therefore, the influence of contact area and number of terms to accuracy is also discussed.
\end{abstract}

\section{Introduction}

Diametrical compression test (also termed as indirect tensile strength test) is a method for measuring the tensile strength of brittles material like rocks. The test is performed by placing a disc-shaped sample (disc thickness twice its diameter) between two rigid platens. Compressive load is applied via steel platen which is in contact with the sample. Since tensile strength of brittle material is much smaller than its compressive strength. Therefore, the brittle material fails under tensile manner, usually in the form of vertical diametrical fracture. The test induces a biaxial state of stress in which the stress at the center of the circular plane is compressive in $x$-direction $\left(\sigma_{x}\right)$, and tensile in $y$-direction $\left(\sigma_{y}\right)$. Theoretically, for an isotropic material, the tensile stress reaches a maximum at a constant magnitude of $P /(\pi a)$, where $P$ represents the applied load and $a$ represents the radius of the circular plane [1].

Several attempts have been seen for modeling. Cauweleart et al. [2] obtained a solution using the theoretical results for a semi-infinite plate under concentrated force. The theoretical solution for orthotropic specimen under concentrated force was shown by Claesson and Bohloli [3], Exadaktylos [4] and Exadaktylos and Kaklis [5]. The theoretical 
solution under uniform radial loading distributed on whole contact area was shown and compared with the result from some experiments by Chen et al. [6] and Markides et al. [7]. Analytical models using finite element were used by Lemmon and Blackketter [8] to evaluate the distribution of tensile stress for diametrical compression test. The contact area between the specimens and two loading platens was set at 2, 4 and $8 \%$ of the circumference of the specimen with uniform loading whose direction is same as loading applied from the platens. The results of this study were in agreement with the theoretical model, using orthotropic stress functions under the same conditions, as proposed by one of authors [9]. The results showed that the tensile stress generating near the loading platens is influenced by the contact area between the loading platens and the specimen. For isotropic materials (i.e., with material properties that are uniform along each axis), the maximum tensile stress at the center of disk gets obvious as the contact area increases. For orthotropic materials, the maximum tensile stress also generates at the center of circular plane when the angle of the principal material direction to the loading direction is close to $\pi / 4$. And the maximum value is almost constant regardless of the contact area. On the other hand, the maximum tensile stress generates near the loading platen decreases as the contact area increases, when the angle between the principal material direction and the loading direction is small. The latter shows that contact area has a significant influence on tensile strength measurements.

In previous studies, the distribution of stress generated on the loading platens was assumed as uniform. In reality, the maximum applied stress seems to generate at the center of contact area, and decreases as the distance from the center increases. Eventually, the applied load seems to disappear at the edges of the contact area. In order to satisfy this boundary condition on the contact area, the cosine curve was adopted as the distribution of stress generated on loading platens in a theoretical model for diametrical loading test by authors. The distribution of tensile stress on the loading radius under this loading is similar to that under uniform loading [10]. However, maximum value of displacement occurring at the center of contact area does not agree with the results from experiments [11]. Furthermore, the hybrid model that consists of uniform loading and the cosine curved loading is applied for the distribution of stress on the contact area [12]. In this model, the ratio of magnitude of the cosine curved loading to that of uniform loading was not estimated correctly.

In this study, the ratio of magnitude of cosine curved loading to that of the uniform loading is re-examined. To calculate with orthotropic stress function by Lekhnitskii [13], resultant forces in $x$ and $y$ direction from this stress distribution should be expanded into Fourier expansion. The accuracy of calculation depending on the number of term is verified.

\section{Fundamental Equations}

In this paper, the problem of the application of oppose concentrated loads $P$ to the diametrical axis of an orthotropic column specimen of radius $a$ is treated as shown in Fig. 1; Here, $\varphi$ is the angle between the loading axis and the principal elastic axis, and $E_{1}$ and $E_{2}$ are the respective elastic moduli in the direction of the principal elastic axis.

By denoting the loading axis as $x$, and the perpendicular direction in the plane of the loading axis as $y$, the following equations representing the resultant forces on surface $X_{n}$ and $Y_{n}$, going anticlockwise from the $x$-axis in the $x$ and $y$ directions, on the boundary circumference of a column-shaped specimen, which are related to stress components $\sigma_{x}, \sigma_{y}$ and $\tau_{x y}$ are obtained:

$$
\left.\begin{array}{l}
X_{n}=\sigma_{x} \cos (n, x)+\tau_{x y} \cos (n, y), \\
Y_{n}=\tau_{x y} \cos (n, x)+\sigma_{y} \cos (n, y) .
\end{array}\right\}
$$




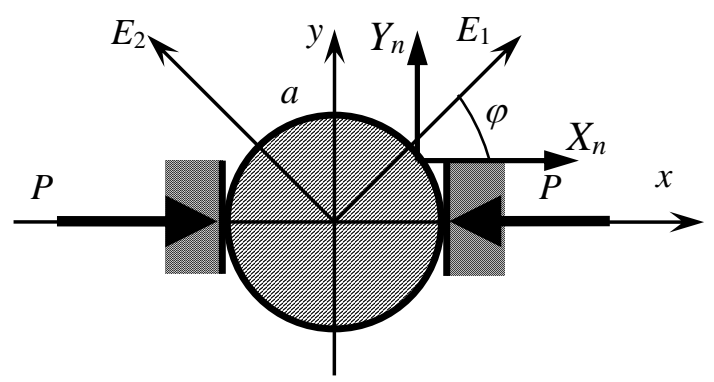

Fig. 1. Orthotropic circular disk under diametrical compression

The stress components $\sigma_{x}, \sigma_{y}$ and $\tau_{x y}$, and the displacement components $u_{x}$ and $u_{y}$ are expressed by the following [13].

$$
\left.\begin{array}{l}
\sigma_{x}=2 \operatorname{Re}\left[\mu_{1}^{2} \Phi_{1}^{\prime}\left(z_{1}\right)+\mu_{2}{ }^{2} \Phi_{2}{ }^{\prime}\left(z_{2}\right)\right], \\
\sigma_{y}=2 \operatorname{Re}\left[\Phi_{1}^{\prime}\left(z_{1}\right)+\Phi_{2}^{\prime}\left(z_{2}\right)\right], \\
\tau_{x y}=-2 \operatorname{Re}\left[\mu_{1} \Phi_{1}^{\prime}\left(z_{1}\right)+\mu_{2} \Phi_{2}^{\prime}\left(z_{2}\right)\right] \cdot
\end{array}\right\}
$$

Here, Re denotes the real part of the complex functions in brackets. ' means the first derivative of $\Phi_{k}\left(z_{k}\right)$ with respect to $z_{k}$.

The integral calculi about resultant forces for the $x$-directions and $y$-directions on the surface are expanded as Fourier series with $M$ terms, as follows:

$$
\left.\begin{array}{c}
\int_{0}^{s} X_{n} d s=\beta_{0}+\sum_{m=1}^{M}\left(\beta_{m} e^{i m \theta}+\bar{\beta}_{m} e^{-i m \theta}\right), \\
-\int_{0}^{s} Y_{n} d s=\alpha_{0}+\sum_{m=1}^{M}\left(\alpha_{m} e^{i m \theta}+\bar{\alpha}_{m} e^{-i m \theta}\right) .
\end{array}\right\}
$$

Here, the bar denotes the complex conjugate. $\alpha_{m}$ and $\beta_{m}$ for the problem in this paper are determined in the next section.

The complex stress functions for an orthotropic circular plate with radius $a$ are expanded as the series expressed below [13]:

$$
\left.\begin{array}{l}
\Phi_{1}\left(z_{1}\right)=A_{0}+A_{1} z_{1}+\sum_{m=2}^{M} A_{m} P_{1 m}\left(z_{1}\right), \\
\Phi_{2}\left(z_{2}\right)=B_{0}+B_{1} z_{2}+\sum_{m=2}^{M} B_{m} P_{2 m}\left(z_{2}\right) .
\end{array}\right\}
$$

Here $P_{k m}\left(z_{k}\right)(k=1,2)$ is a power series of the $m$-th order and is expressed by the following equation. 


$$
\begin{aligned}
P_{k m}\left(z_{k}\right)= & -\frac{1}{a^{m}\left(1-i \mu_{k}\right)^{m}} \\
& \left.\times\left\{\left(z_{k}+\sqrt{z_{k}^{2}-a^{2}\left(1-\mu_{k}^{2}\right.}\right)\right)^{2}+\left(z_{k}-\sqrt{z_{k}^{2}-a^{2}\left(1-\mu_{k}^{2}\right.}\right)^{2}\right\} \quad(k=1,2) .
\end{aligned}
$$

where, $\mu_{k}$ are the roots of the characteristic equation.

$$
a_{11} \mu^{4}-2 a_{16} \mu^{3}+\left(2 a_{12}+a_{66}\right) \mu^{2}-2 a_{26} \mu+a_{22}=0 .
$$

$a_{i j}(i, j=1,2,6)$ represents elastic compliances for plane stress condition. The complex variable $z_{k}$ and power series $P_{k m}\left(z_{k}\right)$ are represented on the cylindrical boundary as follows:

$$
\left.\begin{array}{l}
z_{k}=\frac{a}{2}\left\{\left(1-\mu_{k}\right) e^{i \theta}+\left(1+i \mu_{k}\right) e^{-i \theta}\right\}, \\
P_{k m}=-\left(e^{i m \theta}+t_{k}^{m} e^{-i m \theta}\right), \quad t_{k}=\frac{1+i \mu_{k}}{1-i \mu_{k}} . \quad(k=1,2)
\end{array}\right\}
$$

By using the complex functions $\Phi_{k}\left(z_{k}\right)$, the integral calculi on resultant stresse are expressed as follows:

$$
\left.\begin{array}{rl}
\int_{0}^{s} X_{n} d s & =\operatorname{Re}\left[\mu_{1} \Phi_{1}\left(z_{1}\right)+\mu_{2} \Phi_{2}\left(z_{2}\right)\right], \\
-\int_{0}^{s} Y_{n} d s & =\operatorname{Re}\left[\Phi_{1}\left(z_{1}\right)+\Phi_{2}\left(z_{2}\right)\right]
\end{array}\right\}
$$

By substituting Eq. (8) into Eq. (4), and obtained equations into Eq. (9), the equations for assigning the resultant stress on the boundary are obtained. Furthermore, the coefficients of the corresponding terms of $e^{i m \theta}$ and $e^{-i m \theta}(1 \leq m \leq M)$ are compared with each other, resulting in the following equations:

$$
\begin{aligned}
& A_{1}+B_{1}+\bar{A}_{1}+\quad \bar{B}_{1}=\frac{1}{a}\left(\alpha_{1}+\bar{\alpha}_{1}\right), \\
& \mu_{1} A_{1}+\mu_{2} B_{1}+\bar{\mu}_{1} \bar{A}_{1}+\bar{\mu}_{2} \bar{B}_{1}=\frac{1}{i b}\left(\bar{\alpha}_{1}-\alpha_{1}\right)=\frac{1}{a}\left(\beta_{1}+\bar{\beta}_{1}\right), \\
& \mu_{1}^{2} A_{1}+\mu_{2}^{2} B_{1}+\bar{\mu}_{1}^{2} \bar{A}_{1}+\bar{\mu}_{2}^{2} \bar{B}_{1}=\frac{1}{i b}\left(\bar{\beta}_{1}-\beta_{1}\right) \text {. } \\
& (m=1) \\
& A_{m}+\quad B_{m}+\bar{t}_{1}^{m} \bar{A}_{m}+\bar{t}_{2}{ }^{m} \bar{B}_{m}=-\alpha_{m}, \\
& \mu_{1} A_{m}+\mu_{2} B_{m}+\bar{\mu}_{1} \bar{t}_{1}^{m} \bar{A}_{m}+\bar{\mu}_{2} \bar{t}_{2}{ }^{m} \bar{B}_{m}=-\beta_{m}, \\
& t_{1}^{m} A_{m}+t_{2}{ }^{m} B_{m}+\bar{A}_{m}+\quad \bar{B}_{m}=-\bar{\alpha}_{m}, \\
& \mu_{1} t_{1}^{m} A_{m}+\mu_{2} t_{2}{ }^{m} B_{m}+\bar{\mu}_{1} \bar{A}_{m}+\quad \bar{\mu}_{2} \bar{B}_{m}=-\bar{\beta}_{m} \text {. } \\
& (m \geq 2)
\end{aligned}
$$

For the case of $m=1$, there are only three equations for the four unknown quantities of the real and imaginary parts of $A_{1}$ and $B_{1}$, respectively, so these equations are unsolved. For this reason, the condition that the rigid body rotation is zero is additionally introduced, resulting in the following equation [14]. 


$$
\left(q_{1}-\mu_{1} p_{1}\right) A_{1}+\left(q_{2}-\mu_{2} p_{2}\right) B_{1}+\left(\bar{q}_{1}-\bar{\mu}_{1} \bar{p}_{1}\right) \bar{A}_{1}+\left(\bar{q}_{2}-\bar{\mu}_{2} \bar{p}_{2}\right) \bar{B}_{1}=0 .
$$

where

$$
\left.\begin{array}{ll}
p_{1}=a_{11} \mu_{1}^{2}+a_{12}, & p_{2}=a_{11} \mu_{2}^{2}+a_{12}, \\
q_{1}=\left(a_{12} \mu_{1}^{2}+a_{22}\right) / \mu_{1}, & q_{2}=\left(a_{12} \mu_{2}^{2}+a_{22}\right) / \mu_{2} .
\end{array}\right\}
$$

By adding Eq. (12) to Eq. (10), $A_{1}$ and $B_{1}$ can be obtained completely.

\section{Equations and mathematics}

In this paper, the shape which consists with uniform loading at the center and cosine curved loading at both edges is proposed as distribution of loading on the contact area $\omega / 2$. As shown in Fig. 2, from center of contact area, the uniform loading whose width is $\omega / 8$ are applied on both side. While, from both edge of uniform loading, cosine shaped loading whose width is $\omega / 8$ are applied on both side and loading is disappeared on the edge of contact area. Namely, this means that $50 \%$ of contact area is covered with uniform loading.

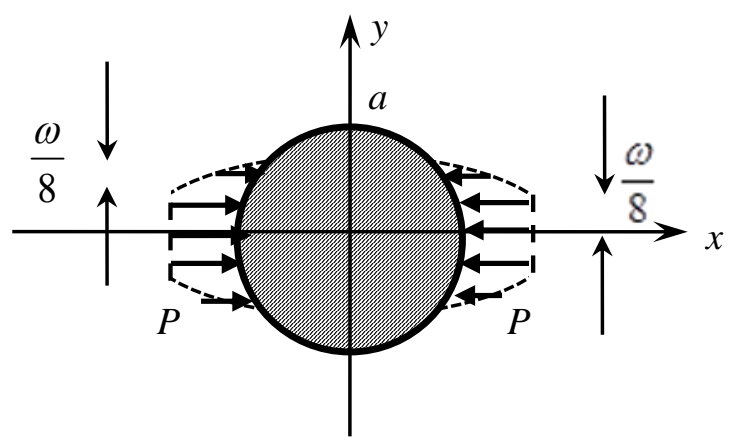

Fig. 2. Boundary condition in this study

Fourier coefficients in Eq. (4) are expressed as shown in Eq. (14) for this problem.

$$
\alpha_{m}=0, \quad \beta_{m}=-i b_{m} \text {. }
$$

$b_{m}$ are able to be obtained through Fourier integral. The first equation in Eq. (4) is an odd function in this problem. Therefore, Fourier sine transform is able to be adopted for this problem. Therefore, the first equation in Eq. (4) is able to be treated as the function between 0 and $\pi$.

Let us define the ratio of magnitude of uniform loading to that of the cosine curved loading. Fig. 3 shows the magnitude of both loading on the half contact area. The magnitudes of uniform loading and cosine curved loading are shown as areas in this figure, $A_{l 1}$ and $A_{l 2}$, respectively. Because contact areas of both loading are same as $\omega / 8$, the ratio of $A_{l 1}$ to $A_{l 2}$ is shown in Eq. (15).

$$
A_{l 1}: A_{l 2}=1: \frac{2}{\pi}
$$

Therefore, the distribution of resultant force in $x$ direction, obtained by integration of $\sigma_{x}$ with $\theta$ in Fig. 3 , is shown in Fig. 4, where $A_{l a}=A_{l 1}+A_{l 2}$.

The distribution of resultant force in Fig. 4 is able to be formulated into Eq. (16); 


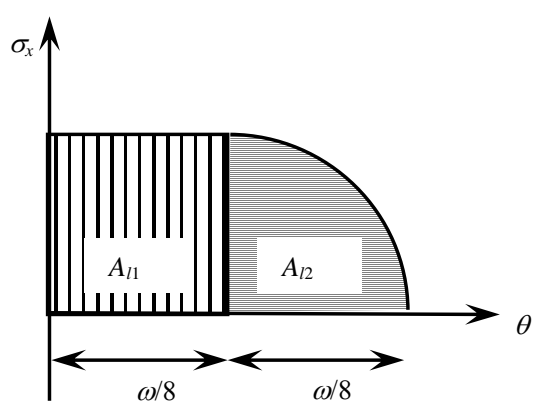

Fig. 3. Magnitude of stress on the loading area

$$
\int X_{n} d s= \begin{cases}\frac{P}{2} \frac{A_{l 1} / A_{l a}}{\omega / 8} \theta, & \left(0 \leq \theta<\frac{\omega}{8}\right) \\ \frac{P}{2} \frac{A_{l 1}}{A_{l a}}-\frac{P}{2} \frac{A_{l 2}}{A_{l a}} \cos \frac{\pi / 2}{\omega / 8} \theta, & \left(\frac{\omega}{8} \leq \theta<\frac{\omega}{4}\right) \\ \frac{P}{2}, & \left(\frac{\omega}{4} \leq \theta<\pi-\frac{\pi}{4}\right) \\ \frac{1}{4}+C_{1} \sin \frac{\pi / 2}{\omega / 8} \theta+C_{2} \cos \frac{\pi / 2}{\omega / 8} \theta, & \left(\pi-\frac{\omega}{4} \leq \theta<\pi-\frac{\omega}{8}\right) \\ \frac{1}{\frac{A_{l 1 /} / A_{l a}}{\omega / 8}(\pi-\theta) .} & \left(\pi-\frac{\omega}{8} \leq \theta<\pi\right)\end{cases}
$$

Where,

$$
C_{1}=-\frac{1}{4} \frac{A_{l 2}}{A_{l a}} \cos \frac{4 \pi}{\omega}\left(\pi-\frac{\omega}{8}\right), \quad C_{2}=\frac{1}{4} \frac{A_{l 2}}{A_{l a}} \sin \frac{4 \pi}{\omega}\left(\pi-\frac{\omega}{8}\right) .
$$

As mentioned before, the distribution of resaltant force is represented using the odd function. Therefore, Fourier coefficients for Eq. (16) is obtained as shown below.

$$
\begin{aligned}
b_{m}=\frac{2 P}{\pi}[ & \int_{0}^{\omega / 8} \frac{4}{\omega} \frac{A_{l 1}}{A_{l a}} \sin m \theta d \theta+\int_{\omega / 8}^{\omega / 4} \frac{1}{2 A_{l a}}\left\{A_{l 1}-A_{l 2} \cos \frac{4 \pi}{\omega} \theta\right\} \sin m \theta d \theta \\
& +\frac{1}{2} \int_{\omega / 4}^{\pi-\omega / 4} \sin m \theta d \theta \\
& +\int_{\pi-\omega / 4}^{\pi-\omega / 8}\left\{\frac{1}{4}+C_{1} \sin \frac{4 \pi}{\omega} \theta+C_{2} \cos \frac{4 \pi}{\omega} \theta\right\} \sin m \theta d \theta \\
& \left.+\int_{\pi-\omega / 8}^{\pi} \frac{4}{\omega} \frac{A_{l 1}}{A_{l a}}(\pi-\theta) \sin m \theta d \theta\right] .
\end{aligned}
$$

The right side of Eq. (18) is calculated into Eq. (19). 


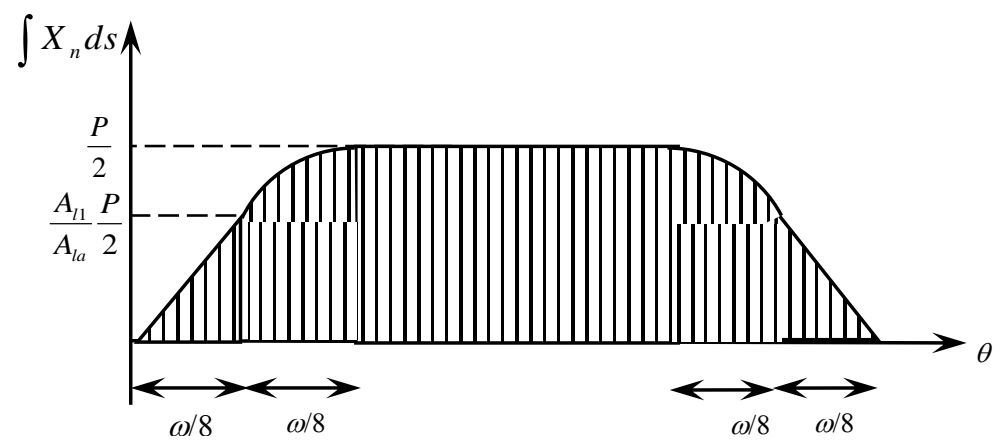

Fig. 4. Distribution of resultant force

$$
\begin{aligned}
b_{m}=\frac{2}{\pi} & \left(B_{1 m}^{*}+B_{2 f m}^{*}+B_{2 b 1 m}^{*}+B_{2 b 2 m}^{*}+B_{3 m}^{*}\right. \\
& \left.+B_{4 f m}^{*}+B_{4 c 1 m}^{*}+B_{4 c 2 m}^{*}+B_{4 b 1 m}^{*}+B_{4 b 2 m}^{*}+B_{5 f m}^{*+}+B_{5 b m}^{*}\right) .
\end{aligned}
$$

where

$$
\left.\begin{array}{l}
B_{1 m}^{*}=-R_{1}\left(\frac{1}{2 m} \cos \frac{m \omega}{8}-\frac{4}{m^{2} \omega} \sin \frac{m \omega}{8}\right), \\
B_{2 f m}^{*}=\frac{R_{1}}{m} \sin \frac{3 m \omega}{16} \sin \frac{m \omega}{16}, \\
B_{2 b 1 m}^{*}=\frac{R_{2}}{4} D_{p m}\left(\cos \frac{\omega \theta_{1 p m}}{4}-\cos \frac{\omega \theta_{1 p m}}{8}\right), \\
B_{2 b 2 m}^{*}=\frac{R_{2}}{4} D_{n m}\left(\cos \frac{\omega \theta_{1 n m}}{4}-\cos \frac{\omega \theta_{1 n m}}{8}\right), \\
B_{3 m}^{*}=\frac{1}{2 m}\left\{\cos \frac{m \omega}{4}-\cos m \theta_{4}\right\}, \\
B_{4 f m}^{*}=\frac{1}{4 m}\left\{\cos m \theta_{4}-\cos m \theta_{8}\right\}, \\
B_{4 c 1 m}^{*}=-\frac{C_{1}}{2} D_{p m}\left\{\sin \theta_{1 p m} \theta_{8}-\sin \theta_{1 p m} \theta_{4}\right\}, \\
B_{4 c 2 m}^{*}=-\frac{C_{1}}{2} D_{n m}\left\{\sin \theta_{1 n m} \theta_{8}-\sin \theta_{1 n m} \theta_{4}\right\}, \\
B_{4 b 1 m}^{*}=-\frac{C_{2}}{2} D_{p m}\left\{\sin \theta_{1 p m} \theta_{8}-\sin \theta_{1 p m} \theta_{4}\right\}, \\
B_{5 b m}^{*}=-\frac{4 R_{1}}{m \omega \pi}\left\{\pi(-1)^{n+1}+\theta_{8} \cos m \theta_{8 m}-\frac{1}{m} \sin m \theta_{8}\right\} . \\
B_{5 f m}^{*}=\frac{4 R_{1}}{m \omega}\left\{(-1)^{m+1}+\cos m \theta_{8}\right\},
\end{array}\right\}
$$




$$
\left.\begin{array}{l}
R_{1}=\frac{A_{l 1}}{A_{l a}}, R_{2}=\frac{A_{l 2}}{A_{l a}}, D_{p m}=\frac{\omega}{m \omega+4 \pi}, D_{n m}=\frac{\omega}{m \omega-4 \pi}, \\
\theta_{1 p m}=m+\frac{4 \pi}{\omega}, \theta_{1 n m}=m-\frac{4 \pi}{\omega}, \theta_{4}=\pi-\frac{\omega}{4}, \theta_{8}=\pi-\frac{\omega}{8} .
\end{array}\right\}
$$

\section{Results and Discussion}

\subsection{Boundary conditions}

In analysis using a series as the present study, the convergence and accuracy must be always discussed. Fig. 5 shows the relation between the integral calculi about resultant force for the $x$-direction along the surface in the longitudinal axis and the number of terms used in Eq. (4) in the transverse axis. Calculation in this figure is carried out for contact area $2 \%, 4 \%$ and $8 \%$. Number of terms $M=20,30,50,70$ and 100 are used in these figures. It is observed that the convergence and the accuracy are improved as number of terms increases for each contact area. It also seems that the convergence is faster as contact area is larger in each case. Fracture happens under contact area from 4.4 to $5.1 \%$ in Tage stone and $6.4 \%$ in cement modified soil [15]. Therefore, calculations are carried out for contact area $4 \%$ and $8 \%$ in this paper and $M=100$ is adopted to obtain the enough accuracy.

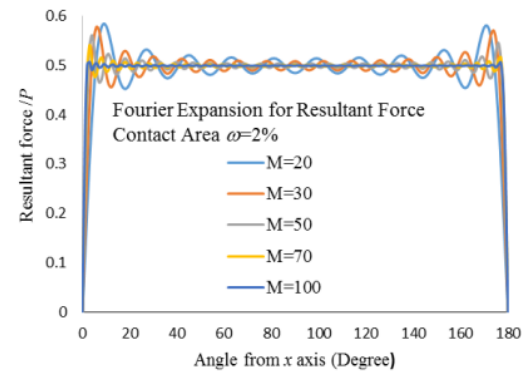

(a) Contact area $2 \%$

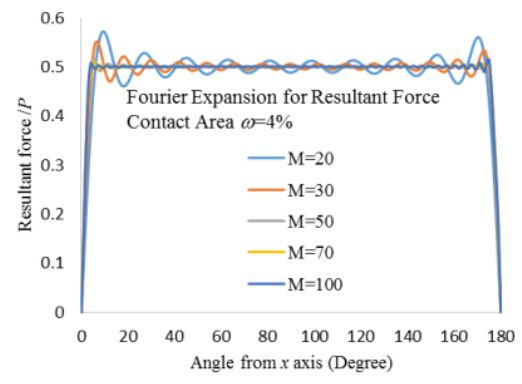

(b) Contact area $4 \%$

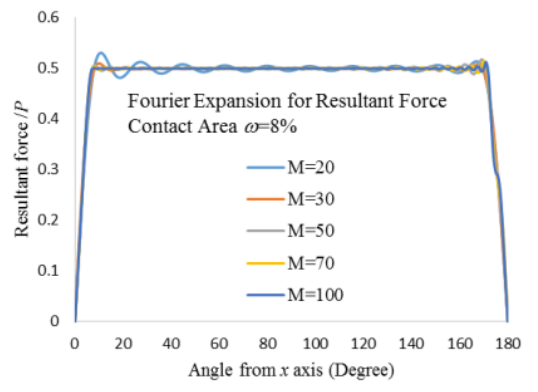

(c) Contact area $8 \%$

Fig. 5. Boundary condition under Case 1

\subsection{Locus of deformation on surface}

Fig. 6 shows the locus of deformation obtained from present result on the surface of disk around loading platen. The locus of deformation is also obtained for uniform loading covering on whole contact area as shown in Fig. 7, and for concentrated force as shown in 
Fig. 8. Fig. 9 shows the locus of deformation obtained under uniform loading covering on whole contact area. These figures show for the both case of contact area $4 \%$ and $8 \%$. Furthermore, Fig. 10 shows the locus of deformation obtained under concentrated force. The locus of deformation is shown at every one degree in the width of 20 degrees from the center of loading platen. The value of locus in each figure is multiplied by $0.05 E_{1} /(\mathrm{Pa})$ to draw. In present results, the shape under loading platen appears flat surface after deformation. In addition, deformation arises towards the center of contact area slightly. While, the maximum value of locus arises at the center of loading platen under uniform loading though it is very small. Under uniform loading, the flat surface area under loading plate is larger than that under the loading proposed in this study. It is also observed that the deformation arises in the loading direction under loading platen. Under concentrated force, the maximum value of locus arises at the center of loading platen and deformation arises toward the center of contact area obviously. In all figures, the dent appears under contact area, namely, displacement in loading direction under the contact area is larger than that around the contact area. However, it is common knowledge that any dents don't appear in actual behavior. Therefore, the results from these calculations are different from the actual behavior of specimen in diametrical loading test using flat loading platens. In these studies, any tangential stresses generating along the loading platen are not taken account into. To improve the accuracy of this study, the theoretical model for tangential stresses generating along the loading platen, like the work by Lavrov [16], will be required.

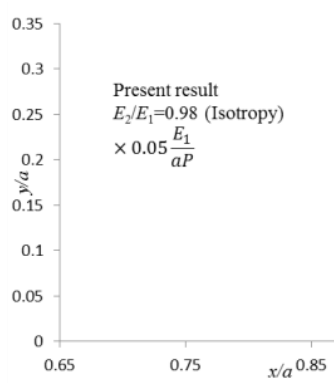

(a) Contact area $4 \%$

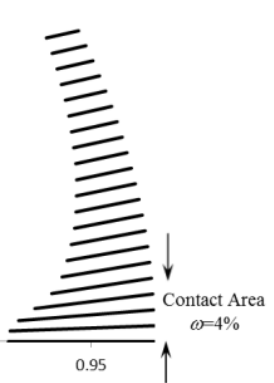

(b) Contact area $8 \%$

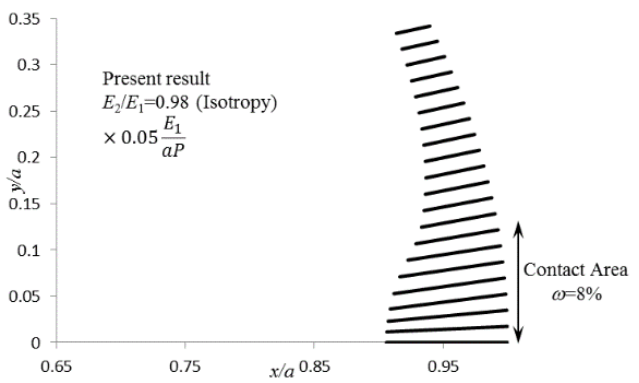

Fig. 6. Locus of deformation on surface in present study

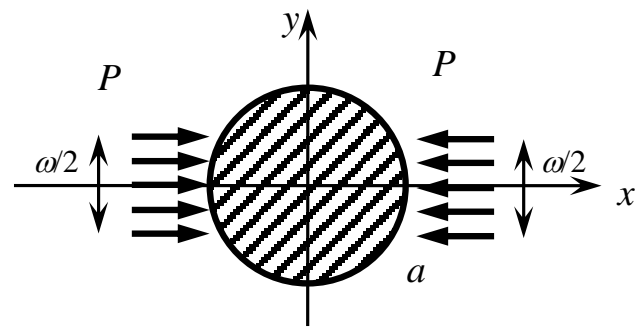

Fig. 7. Scheme of uniform loading on whole contact area

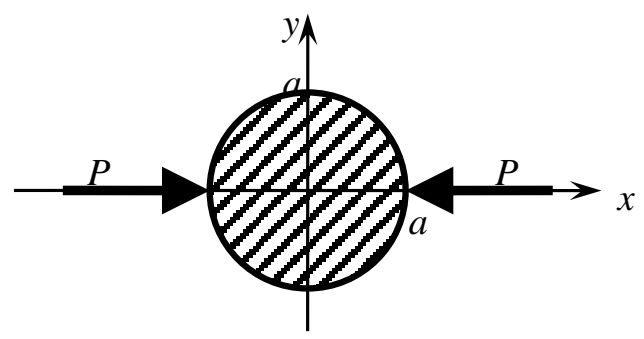

Fig. 8. Scheme of concentrated force 


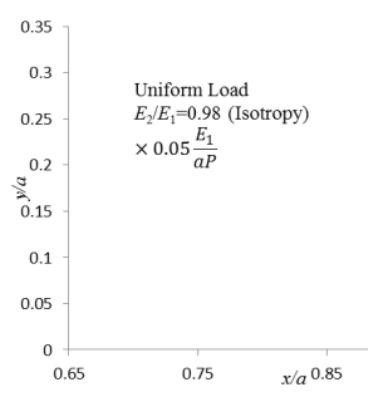

(a) Contact area $4 \%$
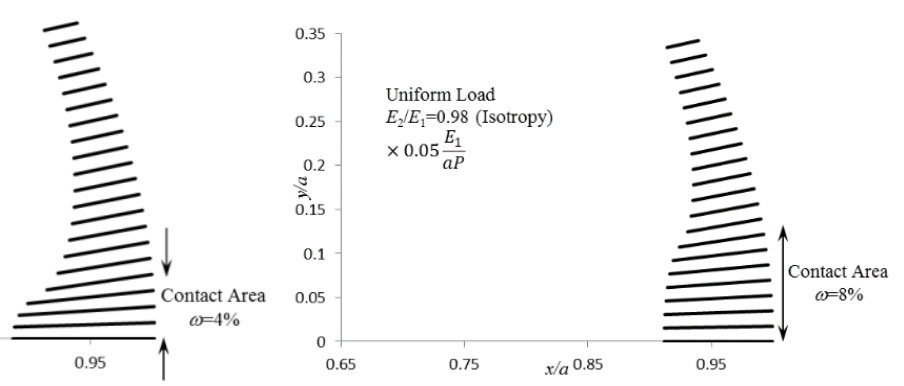

(b) Contact area $8 \%$

Fig. 9. Locus of deformation on surface under uniform loading

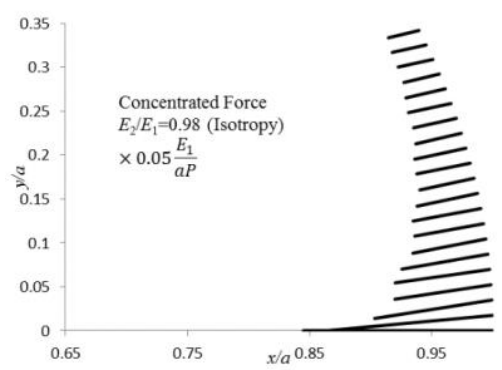

Fig. 10. Locus of deformation on surface under concentrated force

\section{Conclusion}

Previous studies used concentrated force, uniform or cosine shaped loading as boundary conditions for theoretical models for stress distribution in diametrical loading test. The present study adopts combination of loading consists of cosine shape and uniformly distributed loading to satisfy the boundary conditions at the contact area between specimen and loading platens. At first, the boundary condition is verified using the proposed theoretical model and the number of terms in Fourier Series is set to 100 to obtain the adequate accuracy in calculation. Secondly, locus of deformation around the loading platen is shown in isotropic specimen and compared among some results from previous studies. It is shown that almost flat deformation appears along the surface of loading platen and the deformation of surface of specimen under the loading platen appears inside slightly in present study.

In these studies, any tangential stresses along the surface of loading platen or specimen are not taken account into. To establish the theoretical model for these tangential stresses and superpose on the theoretical model proposed in this study are future work for authors.

\section{References}

1. I. S. Sokolnikoff, Mathematical theory of elasticity, McGraw-Hill, 476 (1956).

2. F. V. Cauweleart, and B. Eckmann, Indirect tensile test applied to anisotropic materials, Materials and structures, 27, 54 (1994).

3. J. Claesson, and B. Bohloli, Brazilian test: stress field and tensile strength of anisotropic rocks using an analytical solution, Int. J. of Rock Mech. and Mining Sci., 39, 991 (2002). 
4. G. E. Exadaktylos, On the constraints and relations of elastic constants of transversely isotropic geomaterials, Int. J. of Rock Mech. and Mining Sci., 38, 941 (2001).

5. G. E. Exadaktylos, and K.N. Kaklis, Applications of an explicit solution for the transversely isotropic circular disc compressed diametrically, Int. J. of Rock Mech. and Mining Sci., 38, 227 (2001) .

6. C. S. Chen, , E. Pan and B. Amadei, Determination of Deformability and Tensile Strength of Anisotropic Rock Using Brazilian Tests, Int. J. of Rock Mech. and Mining Sci., 35, 43 (1998).

7. Ch. F. Markides, D. N. Pazis, and S. K. Kourkoulis, Closed full-fill solutions for stress and displacements in the Brazilian disk under distributed radial load, Int. J. of Rock Mech. and Mining Sci., 47, 227 (2010).

8. R. K. Lemmon and D. M. Blackketter, Stress Analysis of an Orthotropic Material under Diametral Compression, Experimental Mech. 36, 204 (1996).

9. T. Tsutsumi, and K. Hirashima, Analysis of Orthotropic Circular Disks and Rings under Diametrical Loading, Structural Eng. and Mech., 9(1), 37 (2000).

10. T. Tsutsumi, and S. Kukino, Distribution of Tensile Stress under Modified Boundary Condition in Theoretical Solution for Diametrical Compression Test, Proc. 13th ISRM Congress, CD-ROM (2015).

11. S. Kukino, and T. Tsutsumi, Modification of boundary condition in theoretical model for diametrical compression test, Proc. 4th International Symposium on Technology for Sustainability, CD-ROM (2014).

12. T. Tsutsumi, R. A. Abdullah, and M. F. M. Amin, Theoretical model using two kinds of function for distribution of applied load in Brazilian Test, Proc. 9th Asian Rock Mechanics Symposium, USB-Flash memory (2016).

13. S. G. Lekhnitskii, Anisotropic Plate, 141, Gordon \& Breach (1968).

14. S. Kawakubo, T. Tsutsumi, and K. Hirashima, Stress and Displacement Fields for an Anisotropic Elliptical Disk Subjected to Arbitrary Loads at Boundary (in Japanese), Trans. JSME Series A, 62, 1626 (1996).

15. Y. Aono, K. Tani, T. Okada, and M. Sakai, The mechanism of failure near the loading point in the splitting tensile strength test on Tage Stone (in Japanese), Proc. of 41th Rock Mech. Symposium in Japan, 157 (2012).

16. Lavrov, A. and Vervoort, A., Theoretical treatment of tangential loading effects on the Brazilian test stress distribution, Int. J. of Rock Mech. and Mining Sci., 39, 275 (2002). 